\title{
Brain Waves as Unconscious Biometrics towards Continuous Authentication - The Effects of Introducing PCA into Feature Extraction -
}

\author{
Isao Nakanishi and Takuya Yoshikawa \\ Graduate School of Engineering \\ Tottori University \\ 4-101 Koyama-minami, Tottori 680-8552, Japan
}

\begin{abstract}
For user management in high-security systems, continuous authentication is required, where unconscious biometrics is suitable. We have proposed to use brain waves as such unconscious biometrics. Assuming continuous authentication of drivers, we have measured brain waves in virtual driving environments and evaluated the verification performance. In this paper, we introduce Principle Component Analysis (PCA) into the feature extraction. It is confirmed that the introduction of PCA is effective for improving the verification performance.
\end{abstract}

Keywords-biometrics; unconscious biometrics; continuous authentication; brain wave; principle component analysis

\section{INTRODUCTION}

From the viewpoint of user management in high-security systems, such as public transport systems which are responsible for many human lives, computer systems which can access to classified information, and distance learning systems in which students receive some qualification or license, one-timeonly authentication is no-good. A registered user gets certified from a system and then he/she switches to another who is not registered to the system, one-time-only authentication cannot prevent such spoofing. On this issue, continuous authentication is the only solution. In addition, only biometrics which can present biometric data unconsciously is applicable in the continuous authentication.

We have proposed to use brain waves as unconscious biometrics [1], [2], [3]. Assuming to apply them to continuous driver authentication, we have measured brain waves in virtual driving environments and evaluated their verification performance. There are many studies which use brain waves as biometrics [4]. However, none of them regards brain waves as unconscious biometrics. They merely treat the brain wave as an alternative of conventional biometrics.

In our conventional approach, the spectra at $\alpha-\beta$ band of brain waves were used as individual features and Support Vector Machine (SVM) was adopted in verification [5]. However, the verification performance obtained was not satisfactory.

In this paper, we try to improve the verification performance by introducing Principle Component Analysis (PCA).

\section{Conventional Approach}

In this section, our conventional approach to person authentication using brain waves is introduced. In the research area using the brain wave as biometrics, it is general to use multiple

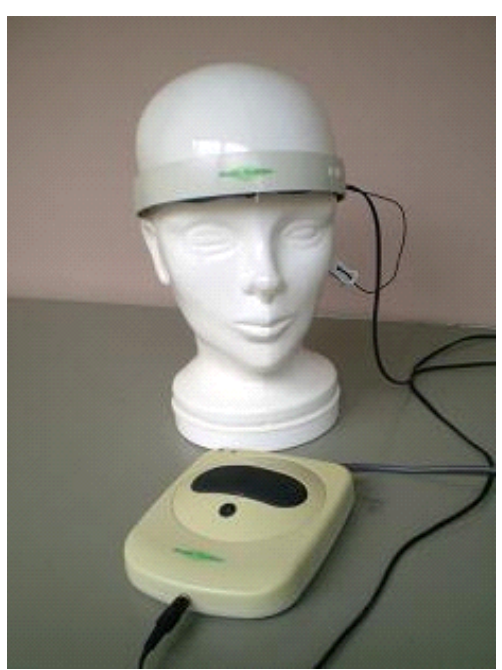

Fig. 1. Brain wave sensor

brain wave sensors to achieve higher verification performance. However, considering practical applications of the brain wave as biometrics, it is inconvenient to set multiple brain wave sensors on scalp while moving hair. On the other hand, we use a brain wave sensor, which is located on the forehead using a headband as shown in Fig. 1. It is relatively acceptable for users to put a sensor on their forehead since the forehead is not relatively covered by hair.

In addition, it is usual to measure brain waves in relaxed and/or eye-closed conditions in the research area. However, it is not realistic to evaluate the verification performance using such brain waves. On the other hand, assuming continuous authentication of drivers in public transport systems, we measured brain waves form thirty subjects in two virtual driving environments, which are named Route Tracing (RT) and simplified Driving Simulator (DS) [3] as shown in Fig. 2.

The subjects wore the brain wave sensor shown in Fig. 1, sat a chair, and watched the screen while doing nothing in RT or controlling the controllers in DS. Please refer to Ref. [3] in details.

The measurement was carried out twice a day and it was repeated five days and so 10 EEGs were obtained from each subject and 120 EEGs were obtained in total. And the spectra 


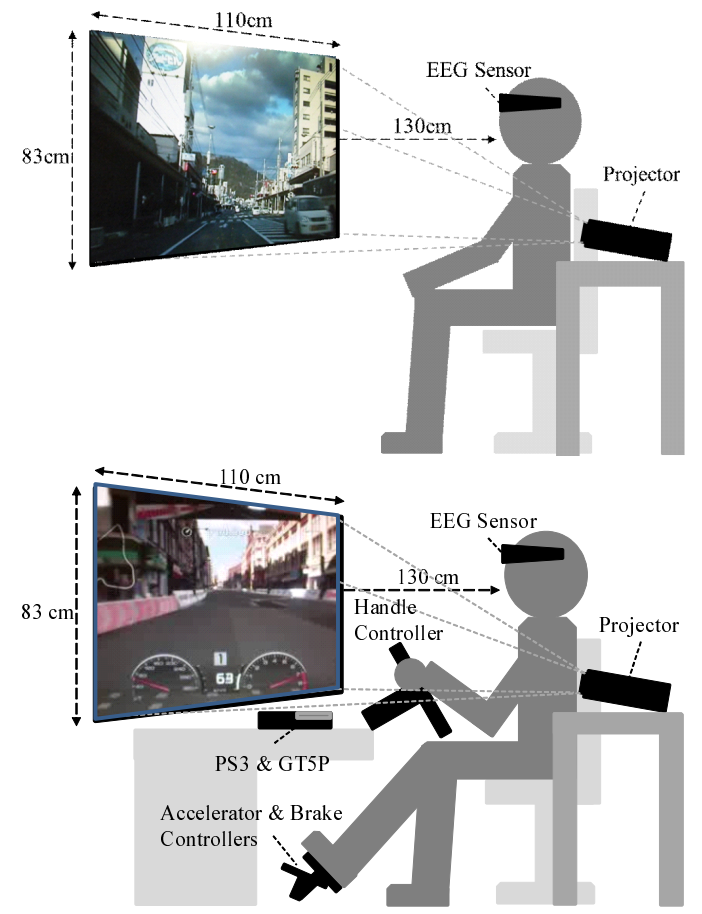

Fig. 2. Measurement of brain waves in virtual driving environments

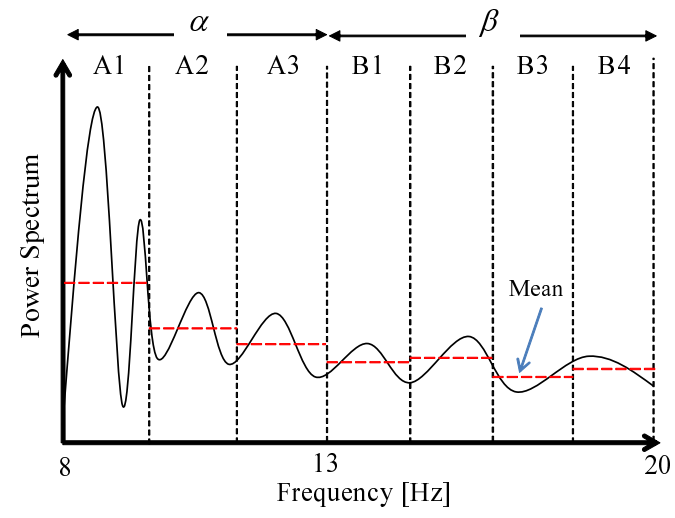

Fig. 3. Spectral means as individual features

of all brain waves measured are calculated using Fast Fourier Transform (FFT) and each spectrum is normalized by dividing each spectral value by the spectral mean. Dividing $\alpha-\beta$ band into several regions and calculating a spectral mean in each region, the spectral means from all regions are extracted as individual features as shown in Fig. 3.

Using SVM as a verification method, we have evaluated the performance of the brain waves. As a result, the verification performance of EER $=30 \%$ was obtained [5] and it is a problem for us to improve the performance.

\section{PRINCIPLE COMPONENT ANALYSIS}

In the conventional approach, the spectra of brain waves are directly used as individual features. In order to improve the verification performance of the brain waves, a new extraction

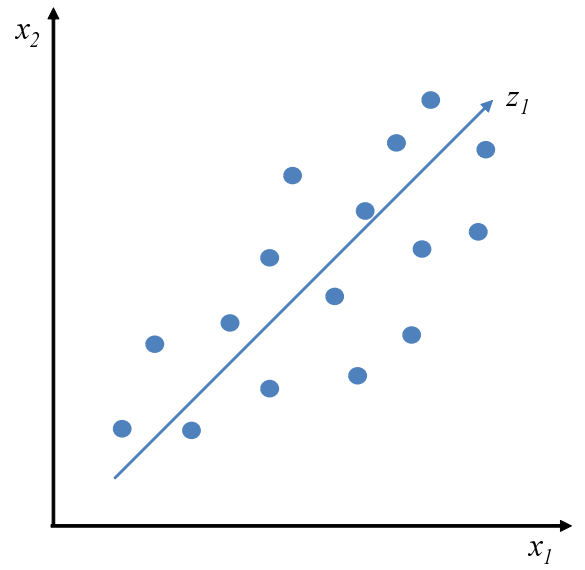

Fig. 4. Distribution of two-variable data

method of individual features is needed. Thus, we introduce PCA into the feature extraction of brain waves.

PCA is one of multivariate analysis methods. Figure 4 shows an example of the distribution of two-variable data. It is clear that there is a relationship between two variables. Thus, an axis which represents the relationship is located as $z_{1}$ in the figure. As a result, we obtain a new axis, that is, a new variable which defines more explicit relationship between the original two variables. $z_{1}$ is a principle component and obtained by seeking a direction on which the variation of data (variance) becomes the largest. To obtain a new variable which has the largest variation of data leads to expanding differences between data; therefore, it is expected to improve verification performance by introducing PCA.

In addition, the original two variables are reduced to one new variable; therefore, the reduction of dimensions can be also achieved by using PCA. If to define only a new axis which obviously represents the relationship between the original two variables is difficult, the second axis which is orthogonally oriented to the first one is defined. In that case, the former is called the first principle component and the latter is the second principle component.

The above explanations are easily extended to a multivariate case. PCA is to present $x_{p}$ with $P$ variables by using $M(\leq P)$ principle components which are independent. $m$ th principle component $z_{m}$ is given by

$$
z_{m}=\sum_{p=1}^{P} w_{p m} x_{p} \quad(m=1,2, \cdots, M)
$$

where $w_{p m}$ are transformation coefficients, which are determined to make the variance of $z_{m}$ in all data the smallest. The result by applying each $x_{p}$ to Eq. (1) is called principal component score.

How each principle component represents the characteristics of original data is defined as the ratio of the variance of each principle component to all variances. It is called contribution ratio. Moreover, the ratio of the variances of $m$ principle components to all variances is called cumulative contribution ratio. In general, the number of principle components used 


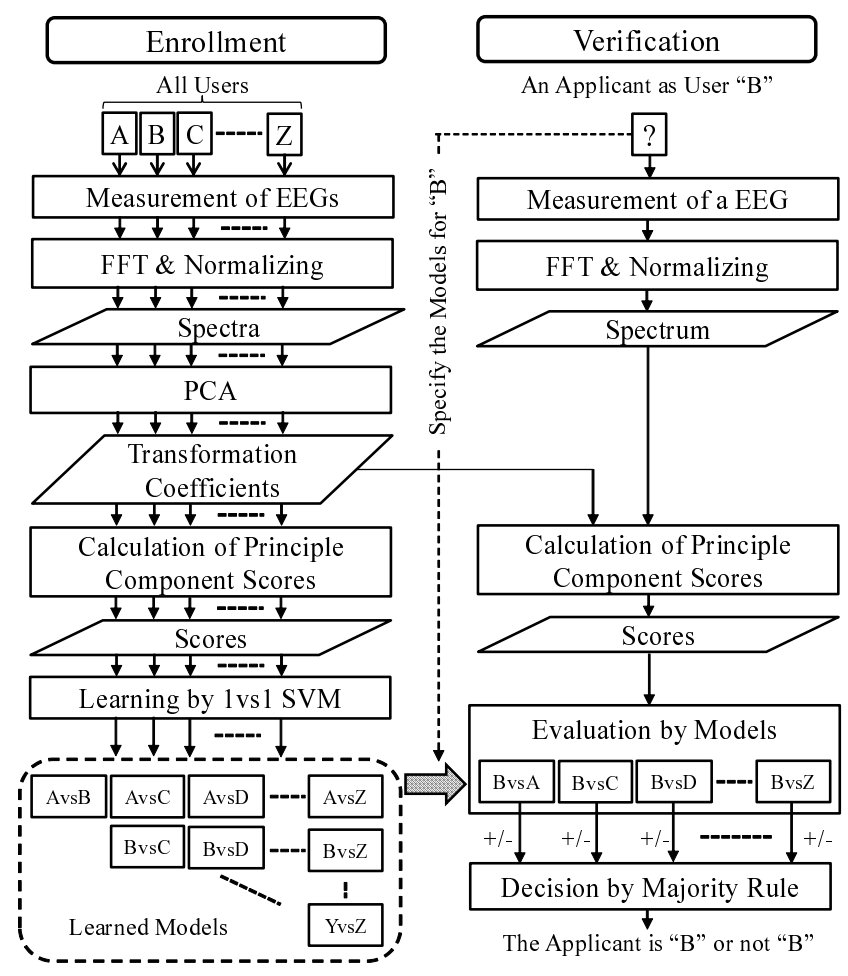

Fig. 5. Verification based on PCA

in analysis is determined to yield the cumulative contribution ratio of about $80 \%$.

\section{VERIFICATION BASED ON PCA}

Figure 5 shows the procedure of the verification method based on PCA. In the enrollment stage, brain waves are measured from all users of a system and then their spectra are obtained by using FFT. After normalizing all spectra, transformation coefficients for each principle component are obtained by applying PCA to the spectra. Next, using the transformation coefficients, a principal component score for each spectrum is calculated. At the same time, using the cumulative contribution ratio, the number of principle components is determined. Principle component scores in the selected principle components are used as individual features. Also, transformation coefficients for the selected principle components are memorized in the system.

Verification is achieved by one versus one Support Vector Machine (1vs1SVM) [6]. SVM is a powerful two-class classifier based on learning. From all users, a pair (two users) is selected, and one is assumed to be a genuine user and another is assumed to be a forger. By giving +1 to the principle component scores of the genuine user and -1 to those of the forger, a SVM model for classifying the two users is learned. In this way, SVM models for all possible pairs are obtained and memorized in the system.

In the verification stage, an applicant who wants to use the system indicates his /her name and specifies a user. Next, his /her brain wave is measured and its spectrum is calculated. Using the spectrum and the transformation coefficients memorized in the system, principle component scores are calculated

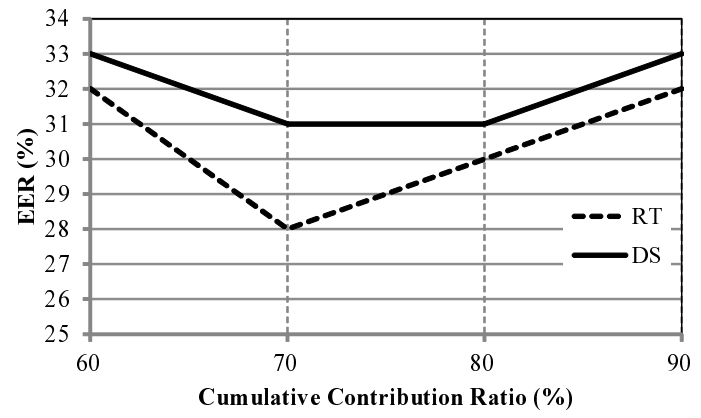

Fig. 6. Relations between EERs and cumulative contribution ratios

and then evaluated in SVM models which are related with the user specified by the applicant. The outputs of the SVM models are real number and so coded to +1 or -1 by using the sign function. When the number of models of which output are +1 is larger than a threshold, the applicant is regarded as genuine.

\section{Performance Evaluation}

In order to evaluate the verification performance of the proposed method, we carried out experimental evaluation using brain waves which had already measured from thirty subjects in two virtual driving environments explained in Sect. II.

In advance of the evaluation, we had to determine the number of principle components which were used in verification. Thus, we examined the verification performance in varying cumulative contribution ratios. The number of learning data was five from each subject. The number of test data was also five from each subject. Optimal kernel functions and parameters for constructing SVM models were found through the grid searching. The verification performance is evaluated using Equal Error Rate (EER), where False Acceptance Rate (FAR) is equal to False Rejection Rate (FRR).

The results are shown in Table I, where the number of

TABLE I. EERS (\%) IN VARIOUS CUMULATIVE CONTRIBUTION RATIOS

\begin{tabular}{c|c|c|c|c}
\hline \multirow{2}{*}{} & \multicolumn{4}{|c}{ Cumulative Contribution Ratio (\%) } \\
\cline { 2 - 5 } & 60 & 70 & 80 & 90 \\
\hline RT & $32(6)$ & $28(12)$ & $30(22)$ & $32(38)$ \\
\hline DS & $33(2)$ & $31(3)$ & $31(10)$ & $33(26)$ \\
\hline
\end{tabular}

principle components is presented in parentheses. These are plotted in Fig. 6. The smallest EER was obtained at the cumulative contribution ratio of $70 \%$ in RT while $70 \%$ and $80 \%$ in DS. From these results, we determined to set the cumulative contribution ratio to $70 \%$.

Next, we evaluated the verification performance. However, the performance of learning-based classifiers generally depends on the number of learning data. Thus, we investigated the verification performances in varying the numbers of learning data. In addition, in order to evaluate the effectiveness of the normalization of spectra which had been adopted in the conventional approach, we tried to remove the normalization from the enrollment and verification stages. 
EERs in various numbers of learning data and with or without the normalization are summarized in Table II, where

TABLE II. EERS (\%) IN VARIOUS NUMBERS OF LEARNING DATA AND WITH/WITHOUT THE NORMALIZATION

\begin{tabular}{c|c|c|c|c}
\hline \multicolumn{2}{c|}{} & \multicolumn{3}{c}{ Number of Learning Data } \\
\cline { 3 - 5 } \multicolumn{2}{c|}{} & 3 & 5 & 7 \\
\hline \multirow{2}{*}{ RT } & withN & 31 & 28 & 25 \\
\cline { 2 - 5 } & outN & 29 & 28 & 22 \\
\hline \multirow{2}{*}{ DS } & withN & 35 & 31 & 26 \\
\cline { 2 - 5 } & outN & 29 & 26 & 24 \\
\hline
\end{tabular}

'withN' and 'outN' mean the cases of with and without normalization, respectively. Additionally, please be aware that the number of test data was five from each subject in the cases of the number of learning data was three and five while the number of test data was three in the case of the number of learning data was seven since the total number of data from each subject was limited to ten.

EERs of the conventional approach were $30 \%$ in both environments [5], in which the number of learning and test data was five. Comparing the conventional EERs with EER of $28 \%$ in RT and $31 \%$ in DS, the effectiveness of introducing PCA is not clear. It could be due to the normalization of spectra. In PCA, principle components are obtained by seeking directions (axes) on which the variations of data (variances) become larger described in Fig. 4. On the other hand, the purpose of introducing the normalization is to reduce the variances of spectra. As a result, the effectiveness of introducing PCA might be not exerted.

EERs without the normalization in RT and DS when the number of learning data was five were $28 \%$ and $26 \%$, respectively. Comparing them with the conventional EERs, the effectiveness of introducing PCA is confirmed. In the case of using PCA, it is better not to use the normalization of spectra.

The relations between the numbers of learning data and EERs are plotted in Fig. 7. From this figure, it is confirmed that EERs were decreased, that is, the verification performances were improved as the number of learning data was increased. As the result, EERs of $22 \%$ in RT and $24 \%$ in DS are achieved. In this evaluation, it was impossible to increase the number of learning data to more than seven since the total number of data was ten. Further improvement of the verification performance might be expected as the number of learning data is increased to ten or more.

\section{COnClusions}

We had studied to use brain waves as biometrics towards continuous authentication. The conventional method had been

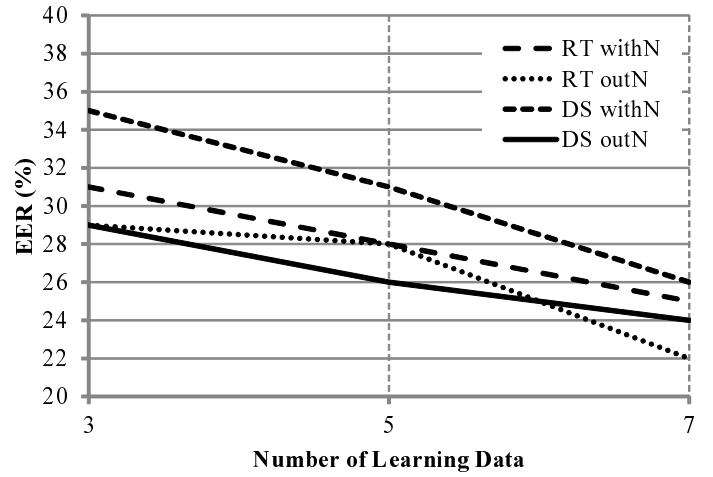

Fig. 7. Relations between EERs and the number of learning data

evaluated using brain waves measured by a single brain wave sensor and in virtual driving environments. In this paper, in order to improve the performance, we introduced PCA into feature extraction. The effectiveness of introducing PCA was confirmed in experimental evaluations. In addition, we also confirmed that it is better not to normalize the spectra of brain waves in the case of using PCA.

On the other hand, the variation of data from each user, that is, the intra-individual variation is not considered when using PCA. In person verification, not only the variation of all data (inter-individual variation) but also the intra-individual variation must be considered for achieving better performance. It is a problem for us to introduce any scheme for reducing intra-individual variations into feature extraction.

\section{REFERENCES}

[1] I. Nakanishi, S. Baba, and C. Miyamoto, "EEG Based Biometric Authentication Using New Spectral Features," Proc. of 2009 IEEE International Symposium on Intelligent Signal Processing and Communication Systems, pp. 651-654, Dec. 2009.

[2] I. Nakanishi, C. Miyamoto, and S. Li, "Brain Waves as Biometrics in Relaxed and Mental Tasked Conditions with Eye-Closed," International Journal of Biometrics, vol. 4, no. 4, pp. 357-372, 2012.

[3] I. Nakanishi, S. Baba, K. Ozaki, and S. Li, "Using Brain Wave as Transparent Biometrics for On-Demand Driver Authentication," International Journal of Biometrics, vol. 5, nos. 3-4, pp. 321-335, 2013.

[4] M. Pozo-Banos, J. Alonso, J. Ticay-Rivas, and C. Travieso, "Electroencephalogram Subject Identification: A Review," Expert Systems with Applications, vol. 41, pp. 6537-6554, 2014.

[5] T. Yoshikawa, H. Fukuda, I. Nakanishi and S. Li, "Person Authentication using EEG - Verification Based on 1vs1 SVM with Divided EEG Spectra -," Proc. of the 2013 International Workshop on Smart Info-Media Systems in Asia, pp. 367-371, Oct, 2013.

[6] C. Hsu and C. Lin, "A Comparison of Methods for Multiclass Support Vector Machines," IEEE Trans. Neural Networks, vol. 13, no. 2, pp. 415-425, 2002. 